OPEN ACCESS

Edited by: Andrej Wagner,

Paracelsus Medical University, Austria

Reviewed by:

Piotr G. Rychahou,

University of Kentucky, United States

Lowell Anthony,

University of Kentucky, United States

*Correspondence:

Yan-Shen Shan

ysshan@mail.ncku.edu.tw

${ }^{+}$These authors have contributed equally to this work and

share first authorship

Specialty section:

This article was submitted to

Gastrointestinal Cancers: Hepato

Pancreatic Biliary Cancers,

a section of the journal

Frontiers in Oncology

Received: 14 July 2021 Accepted: 28 October 2021 Published: 12 November 2021

Citation:

Tsai H-J, Hsiao C-F, Chang JS

Chen L-T, Chao Y-J, Yen C-J and

Shan Y-S (2021) The Prognostic and

Predictive Role of Chromogranin

A in Gastroenteropancreatic

Neuroendocrine Tumors -

A Single-Center Experience.

Front. Oncol. 11:741096

doi: 10.3389/fonc.2021.741096

\section{The Prognostic and Predictive Role of Chromogranin A in Gastroenteropancreatic Neuroendocrine Tumors - A Single-Center Experience}

\author{
Hui-Jen Tsai ${ }^{1,2,3 t}$, Chin-Fu Hsiao ${ }^{4 \dagger}$, Jeffrey S. Chang ${ }^{1}$, Li-Tzong Chen ${ }^{1,2,3}$, Ying-Jui Chao ${ }^{5}$ \\ Chia-Ju Yen ${ }^{2}$ and Yan-Shen Shan ${ }^{5,6 *}$ \\ ${ }^{1}$ National Institute of Cancer Research, National Health Research Institutes, Tainan, Taiwan, ${ }^{2}$ Department of Oncology, \\ National Cheng Kung University Hospital, College of Medicine, National Cheng Kung University, Tainan, Taiwan, \\ ${ }^{3}$ Department of Internal Medicine, Kaohsiung Medical University Hospital, Kaohsiung, Taiwan, ${ }^{4}$ Institute of Population Health \\ Sciences, National Health Research Institutes, Zhunan, Taiwan, ${ }^{5}$ Division of General Surgery, Department of Surgery, \\ National Cheng Kung University Hospital, College of Medicine, National Cheng Kung University, Tainan, Taiwan, 6 Institute of \\ Clinical Medicine, College of Medicine, National Cheng Kung University, Tainan, Taiwan
}

Chromogranin A ( $\mathrm{CgA})$ is a non-specific biomarker excreted by neuroendocrine tumor (NET) cells. Elevation of circulating CgA level can be detected in gastroenteropancreatic (GEP)-NET patients and has been shown to correlate with tumor burden. The prognostic and predictive roles of $\mathrm{CgA}$ level and the change of $\mathrm{CgA}$ level are controversial. In this study, we retrospectively analyzed 102 grade 1/2 GEP-NET patients with available baseline or serial follow-up CgA levels from the National Cheng Kung University Hospital to evaluate the association between circulating $\mathrm{CgA}$ level and the tumor extent, overall survival (OS), and tumor response prediction. The baseline characteristics, baseline CgA level, and change of CgA level during follow-up and their association was analyzed. Sixty cases had baseline CgA levels available prior to any treatment and ninety-four cases had serial follow-up CgA levels available during treatment or surveillance. Baseline CgA levels were associated with stage and sex. Higher baseline CgA levels were associated with worse OS after adjusting for sex, stage, grade, primary site, and functionality (hazard ratio $=13.52,95 \%$ confidence interval $(C l), 1.06-172.47$, $P=0.045)$. The cross-sectional analysis for the change of $\mathrm{CgA}$ level during follow-up showed that $a \geq 40 \%$ increase of $\mathrm{CgA}$ meant a higher probability of developing tumor progression or recurrence than those with a $<40 \%$ increase of $\mathrm{CgA}$ level (odds ratio $=5.04$, 95\% Cl, 1.31-19.4, $P=0.019$ ) after adjusting for sex, age, grade, stage, and functionality. Our study results suggest that CgA may be a predictive marker for tumor burden, OS, and tumor progression in GEP-NET patients.

Keywords: chromogranin A, neuroendocrine tumors, gastroenteropancreas, prognostic factor, predictive factor 


\section{INTRODUCTION}

Neuroendocrine tumors (NETs) are relatively rare neoplasms with neuroendocrine differentiation. NETs may arise from anywhere in the body with gastroentero-pancreatic (GEP) sites being the most common origins of NETs (1-3). The incidence of NETs has been increasing rapidly in recent decades $(1-5)$. NETs are divided into two categories, functioning and non-functioning tumors. Functioning tumors secret substances causing specific symptoms. The substances produced by non-functioning tumors do not cause specific symptoms (6). The presence of specific symptoms may facilitate early diagnosis of NETs. For example, NETs excreting insulin (insulinoma) may induce hypoglycemia and cause dizziness, weakness, sweating, or consciousness disturbance that can be easily identified and diagnosed at an early stage. However, early diagnosis of non-functioning tumors is difficult due to the lack of specific symptoms. Because NETs are of a heterogeneous nature, the secreted substances differ among different NETs. The specific substances secreted by functioning GEP-NETs include insulin, glucagon, gastrin, serotonin, somatostatin, and vasoactive intestinal peptide (6). However, most tumor cells produce non-specific substances, such as chromogranin A ( $\mathrm{CgA})$ and neuro-specific enolase (NSE). These circulating biomarkers may play a diagnostic, prognostic, or predictive role for GEP-NETs; however, there are also limitations noted for these biomarkers (6).

$\mathrm{CgA}$ is a member of granins, which are abundantly distributed in endocrine, neuroendocrine, and immune cells. $\mathrm{CgA}$ can be proteolytically cleaved into biologically active peptides, such as vasostatin, pancreastatin, catestatin, and serpinins, by various enzymes, such as prohormone convertases, cathepsin L, plasmin, and kallikrein. CgA and its fragments can be detected in the blood of patients of various non-cancer diseases, such as heart failure, hypertension, thyroid disease, renal failure, liver disease, inflammatory bowel disease, rheumatoid disease, and cancers. They may also play roles in cardiovascular, immunometabolic, and cancer regulation (7-9). CgA is the most commonly used circulating biomarker for NETs in clinical practice. The sensitivity of circulating $\mathrm{CgA}$ is considered acceptable for the diagnosis of functional and advanced NETs whereas the specificity of circulating $\mathrm{CgA}$ for the diagnosis of NETs is not ideal. In addition to non-cancer disease, many other factors may interfere with the $\mathrm{CgA}$ level, such as food and drugs. $\mathrm{CgA}$ level can also be elevated in other non-NET cancers, such as breast cancer, thyroid cancer, pancreatic cancer, hepatocellular carcinoma, gastric cancer, colon cancer, and prostate cancer $(8,9)$. Therefore, the use of circulating CgA in diagnosis or screening has been limited. Nevertheless, circulating $\mathrm{CgA}$ has been commonly used for the follow-up of NETs (7-9). CgA level has been associated with the disease extent of GEP-NETs (10-12). Circulating CgA has been shown to be a biomarker for predicting survival and treatment response in advanced NET patients, but fewer studies were conducted for early-stage NETs (13-16). We retrospectively identified grade 1 (G1) and grade 2 (G2) GEP-NET patients with available baseline CgA or with serial follow-up CgA levels to correlate CgA levels with the clinical characteristics and outcomes.

\section{PATIENTS AND METHODS}

\section{Patient Identification and Data Collection}

Patients diagnosed with G1 or G2 GEP-NETs at the National Cheng Kung University Hospital and with CgA levels available were included in this study. The data of patients' demographic characteristics, including sex and age at diagnosis, and clinical information, including grade, stage, primary site, and functionality of the tumor, baseline $\mathrm{CgA}$ levels, serial follow-up of CgA levels during treatment or surveillance, treatment response, and survival status were collected by chart review. The study protocol was reviewed and approved by the Institutional Review Board of National Cheng Kung University Hospital. Because this study is a retrospective review of the chart, no informed consent is needed.

\section{Evaluation of Response and Survival}

The patients received serial image examination for evaluation of disease status. The advanced-stage patients who received systemic treatment had serial image follow-ups every 2 to 6 months. The early-stage patients who had curative resection of the tumors had serial image follow-ups every 3-12 months. Tumor response was evaluated retrospectively according to the Response Evaluation Criteria In Solid Tumors (RECIST) v1.1. Overall survival (OS) was defined as the time from the diagnosed date to death or the last follow-up date.

\section{Evaluation of CgA level}

The CgA levels were measured every 1-12 months for the patients. The frequency of $\mathrm{CgA}$ measurement was determined by the disease status and duration of each visit. The measurement frequency of CgA levels was every 1 to 3 months for the advanced-stage patients under treatment. The measurement frequency of CgA levels was every 3 to 12 months for the patients who had received curative resection without residual disease. The blood samples were sent to the Union Clinical Laboratory (Taipei, Taiwan) for the measurement of CgA levels. An automated immunofluorescent assay was used to detect the CgA levels by using Kryptor, Brahms. The baseline CgA level was defined as the CgA level detected prior to any treatment of NET. A CgA level more than 2-fold the upper normal limit of CgA $(101.9 \mathrm{ng} / \mathrm{ml})$ was defined as high whereas a CgA level less than 2 -fold the upper normal limit of CgA was defined as low. The change of $\mathrm{CgA}$ level $(\triangle \mathrm{CgA})$ was determined by subtracting the first CgA level detected prior to or during the treatment of NET from the last $\mathrm{CgA}$ level detected prior to the disease progression or recurrence. The value of $\Delta \mathrm{CgA}$ divided by the first $\mathrm{CgA}$ level detected prior to or during the treatment of NET was defined as the ratio of change of CgA.

\section{Statistical Analysis}

All statistical analyses were performed using SAS statistical software (Version 8.2, SAS Institute Inc., Cary, NC, U.S.A). Summary statistics, including mean and standard deviation, were provided for continuous variables. Frequencies and proportions were used to summarize categorical data. The differences between high and low baseline CgA levels in NET 
patients were analyzed by Pearson's chi-square test or exact test for variables including sex, grade, functionality, stage, and primary site. The relationship between OS and the potential explanatory factors was determined using the Cox proportional hazards model. In addition, the survival probabilities were all estimated by the Kaplan-Meier method for each group. Logistic regression analyses were performed to assess the effects of variables on the risk of progressive disease (PD) events. All tests were two-tailed. A p value $<0.05$ was considered significant.

\section{RESULTS}

\section{Patient Characteristics}

There were 102 patients diagnosed with G1 or G2 GEP-NETs at the National Cheng Kung University Hospital from 2008 to 2020 with baseline CgA or serial follow-up CgA levels available. The patient characteristics are listed in Table 1. The mean age of all patients was 53.7 (range: 18-82) years old. There were 55 (53.9\%) men and $47(46.1 \%)$ women. Sixty-three $(61.8 \%)$ cases were G1 and thirty-nine (38.2\%) cases were G2. The percentages of stages I, II, III, and IV of the cases were $47.1 \%, 17.6 \%, 7.8 \%$, and $27.5 \%$, respectively. According to the site, 68, 9, 8, and 7 cases were located in the pancreas, stomach, rectum, and duodenum, respectively. The other cases included NETs of ampulla of Vater $(\mathrm{N}=4)$, liver $(\mathrm{N}=3)$, colon $(\mathrm{N}=2)$, and appendix $(\mathrm{N}=1)$. Thirty (29.4\%) cases were functioning tumors. Among these 102 cases, 60 cases had baseline CgA levels available prior to any treatment and 94 cases had serial follow-up of CgA levels available before and after treatment for further analysis.

TABLE 1 | Baseline characteristics.

\begin{tabular}{lc}
\hline & $\mathbf{N}=\mathbf{1 0 2}(\mathbf{\%})$ \\
\hline Age at diagnosis & \\
Mean \pm std & $53.7 \pm 14.3$ \\
Median, range & $54,18-82$ \\
Sex & \\
F & $47(46.1 \%)$ \\
M & $55(53.9 \%)$ \\
Grade & \\
G1 & $63(61.8 \%)$ \\
G2 & $39(38.2 \%)$ \\
Stage & \\
I & $48(47.1 \%)$ \\
II & $18(17.6 \%)$ \\
III & $8(7.8 \%)$ \\
IV & $28(27.5 \%)$ \\
Primary site & \\
Pancreas & $68(66.7 \%)$ \\
Non-pancreas & $34(33.3 \%)$ \\
Stomach & 9 \\
Rectum & 8 \\
Duodenum & 7 \\
Ampulla of Vater & 4 \\
Liver, colon, appendix & $3,2,1$ \\
Functionality & \\
No & $72(70.6 \%)$ \\
Yes & $30(29.4 \%)$ \\
\end{tabular}

\section{Baseline CgA Level Was Associated With OS of NET Patients}

The distributions of the 60 cases with available baseline $\mathrm{CgA}$ levels by various variables, including sex, primary site, grade, stage, and functionality of the tumor are shown in Table 2 . The high or low baseline CgA levels in NET patients were not associated with the primary site, grade, and functionality of the tumors. A higher proportion of women had high baseline $\mathrm{CgA}$ levels than the men in our NET patients $(P=0.045)$. The distribution of baseline CgA levels was associated with stage $(P=0.023)$. Stage I patients had a lower percentage with high baseline CgA levels (low versus high, 21:6) than stage IV patients (low versus high, 12:10).

We analyzed the association between baseline CgA levels and the OS of the NET patients (Table 3). The Kaplan Meier survival curves of the patients are shown in Figure 1. The OS was better in the patients with low baseline CgA levels. The survival rate in the patients with low baseline CgA levels was $97.4 \%$ whereas the survival rate in the patients with high baseline CgA levels was 68.2\% $(P=0.001)$. We further analyzed the association between OS and baseline CgA level by sex, primary site, grade, stage, and functionality of the tumors. The significant association between OS and baseline CgA levels in subgroup analysis was still present as shown in Table 3. Men with low baseline CgA levels had better OS than men with high baseline CgA levels (survival rate 100\% vs $62.5 \%, P=0.001)$. The difference was not observed in women (survival rate $92.9 \%$ vs $71.4 \%, P=0.126$ ). The patients with low baseline CgA levels had significantly better OS in pancreatic NETs (survival rate $96.6 \%$ vs $57.1 \%, P=0.0002$ ) but not in nonpancreatic NETs (survival rate $100 \%$ vs $87.9 \%, P=0.317$ ). The OS was significantly better in G2 (survival rate $92.3 \%$ vs $45.5 \%$, $P=0.007)$ patients with low baseline CgA levels than those with high baseline CgA level but the difference was not observed in G1 (survival rate $100 \%$ vs $90.9 \%, P=0.145$ ) patients. The OS was high in G1 patients irrelevant of the baseline CgA levels. The OS was better for patients with functioning tumors who had low baseline CgA levels than those with high baseline CgA levels (survival rate $100 \%$ vs $63.6 \%, P=0.004)$. However, the OS was not significantly different in the NET patients with non-functioning tumors irrespective of their baseline CgA levels $(P=0.098)$. The association between $\mathrm{OS}$ and the baseline CgA levels was significantly different in patients with stage I/II or III/IV tumors. The OS was better for the patients with stage I/II tumors who had low baseline CgA levels than those with high baseline CgA levels (survival rate $100 \%$ vs $81.8 \%, P=0.034$ ). The survival rate of the stage III/IV patients with low baseline CgA levels was $93.8 \%$ but the survival rate of those with high baseline CgA levels was only 54.6\% $(P=0.019)$. The Cox proportional hazard ratio analysis for OS was performed by baseline $\mathrm{CgA}$ level, sex, age, grade, stage, and functionality (Table 4). Because there were fewer case numbers in stage II and III, we analyzed stage I and II combined versus stage III and IV combined. In the univariate analysis, patients with high baseline CgA levels had a worse OS than those with low baseline CgA levels (hazard ratio $(\mathrm{HR})=14.31,95 \%$ confidence interval $(\mathrm{CI}): 1.76-116.48)$. The G2 patients had a worse OS than those of G1 $(\mathrm{HR}=11.71,95 \% \mathrm{CI}$ : 
TABLE 2 | The distribution of baseline CgA level in GEP-NETs.

\begin{tabular}{|c|c|c|c|}
\hline & $\begin{array}{c}\text { Low } \\
N=38\end{array}$ & $\begin{array}{c}\text { High } \\
N=22\end{array}$ & $P^{\star}$ value \\
\hline Sex & & & 0.045 \\
\hline Men & $24(63.2 \%)$ & 8 (36.4\%) & \\
\hline Women & 14 (37.8\%) & $14(63.6 \%)$ & \\
\hline Grade & & & 0.229 \\
\hline G1 & 25 (65.8\%) & $11(50 \%)$ & \\
\hline G2 & 13 (34.2\%) & $11(50 \%)$ & \\
\hline Functionality & & & 0.428 \\
\hline Y & 15 (39.5\%) & $11(50 \%)$ & \\
\hline $\mathrm{N}$ & 23 (60.5\%) & $11(50 \%)$ & \\
\hline Stage & & & 0.023 \\
\hline I & $21(55.3 \%)$ & $6(27.3 \%)$ & \\
\hline$\|$ & 1 (2.6\%) & 5 (22.7\%) & \\
\hline III & 4 (10.5\%) & $1(4.5 \%)$ & \\
\hline IV & 12 (31.6\%) & $10(45.5 \%)$ & \\
\hline Primary site & & & 0.294 \\
\hline Pancreas & 29 (76.3\%) & 14 (63.6\%) & \\
\hline Non-pancreas & 9 (23.7\%) & $8(36.4 \%)$ & \\
\hline
\end{tabular}

${ }^{*}$ Chi-square test or exact test.
1.44-95.39). In multivariate analysis, significantly worse OS persisted for the patients with high baseline CgA levels versus low baseline $\mathrm{CgA}$ levels ( $\mathrm{HR}=13.52,95 \% \mathrm{CI}: 1.06-172.47)$ and for the patients of G2 NET versus G1 NET (HR $=41.81,95 \%$ CI: 1.68-1041.72). The OS of the GEP-NET patients did not differ by age, sex, stage, and functionality.

\section{The Change of CgA Level May Be a Predictor for Tumor Progression}

Ninety-four cases had serial follow-up of CgA levels available during the follow-up. The $\Delta \mathrm{CgA}$ for each patient was calculated. For the patients without $\mathrm{PD}$, including complete response, partial response or stable disease, or without tumor recurrence, the $\Delta \mathrm{CgA}$ was the value of the last $\mathrm{CgA}$ level detected prior to the last evaluation of tumor response subtracted by the first $\mathrm{CgA}$ level before or during treatment or surveillance of NET. The ratio of $\triangle \mathrm{CgA}$ divided by the first CgA level before or during treatment or surveillance of NET was calculated. The cut off value of the ratio of change of CgA levels in the NET patients was 0.4 based on the significance of correlation

TABLE 3 | The overall survival rate of NET patients with low or high baseline CgA levels.

\begin{tabular}{|c|c|c|c|c|}
\hline Baseline CgA level & Case number (N) & Survival rate (\%) & Median survival (years) & $P^{\star}$ value \\
\hline All & & & & 0.001 \\
\hline Low & 38 & 97.4 & - & \\
\hline High & 22 & 68.2 & - & \\
\hline Baseline CgA level by sex & & & & 0.009 \\
\hline Men & 32 & & & 0.001 \\
\hline Low & 24 & 100 & - & \\
\hline High & 8 & 62.5 & - & \\
\hline Women & 28 & & & 0.126 \\
\hline Low & 14 & 92.9 & - & \\
\hline High & 14 & 71.4 & - & \\
\hline Baseline CgA level by primary site & & & & 0.0002 \\
\hline Pancreas & 43 & & & 0.0002 \\
\hline Low & 29 & 96.6 & - & \\
\hline High & 14 & 57.1 & - & \\
\hline Non-pancreas & 17 & & & 0.317 \\
\hline Low & 9 & 100 & - & \\
\hline High & 8 & 87.9 & - & \\
\hline Baseline CgA level by grade & & & & $<0.0001$ \\
\hline G1 & 36 & & & 0.145 \\
\hline Low & 25 & 100 & - & \\
\hline High & 11 & 90.9 & - & \\
\hline G2 & 24 & & & 0.007 \\
\hline Low & 13 & 92.3 & - & \\
\hline High & 11 & 45.5 & 1.7 & \\
\hline Baseline CgA level by stage & & & & 0.002 \\
\hline I/II & 33 & & & 0.034 \\
\hline Low & 22 & 100 & - & \\
\hline High & 11 & 81.8 & - & \\
\hline III/IV & 27 & & & 0.019 \\
\hline Low & 16 & 93.8 & - & \\
\hline High & 11 & 54.6 & - & \\
\hline Baseline CgA level by functionality & & & & 0.004 \\
\hline Functioning & 26 & & & 0.004 \\
\hline Low & 15 & 100 & - & \\
\hline High & 11 & 63.6 & - & \\
\hline Non-functioning & 34 & & & 0.098 \\
\hline Low & 23 & 95.7 & - & \\
\hline High & 11 & 72.7 & - & \\
\hline
\end{tabular}

*Log-rank test. 

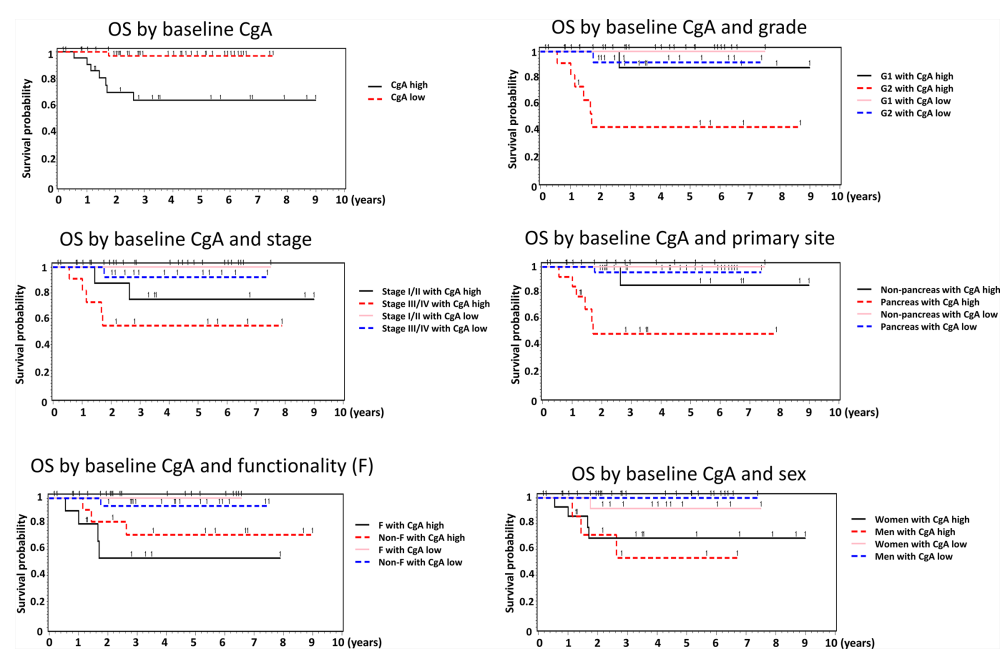

FIGURE 1 | The Kaplan Meier survival curves of the GEP-NET patients by baseline CgA level in the overall patients and by sex, grade, stage, primary site, and functionality of the tumors.

TABLE 4 | The Cox proportional analysis for OS of NETs by baseline CgA level, sex, age, grade, stage, and functionality.

\begin{tabular}{|c|c|c|c|c|c|c|}
\hline & \multicolumn{2}{|c|}{ Univariate } & \multirow[t]{2}{*}{$P^{*}$ value } & \multicolumn{2}{|c|}{ Multivariate } & \multirow[t]{2}{*}{$P^{\star}$ value } \\
\hline & HR & $95 \% \mathrm{Cl}$ & & HR & $95 \% \mathrm{Cl}$ & \\
\hline Baseline CgA level, high vs low & 14.3 & $1.76-116.48$ & 0.013 & 13.52 & $1.06-172.47$ & 0.045 \\
\hline Age of diagnosis & 1.04 & 0.99-10.9 & 0.160 & 1.08 & 0.98-1.19 & 0.101 \\
\hline Sex, men vs women & 0.51 & $0.12-2.14$ & 0.358 & 2.07 & $0.24-17.83$ & 0.507 \\
\hline Grade, G2 vs G1 & 11.71 & $1.44-95.39$ & 0.022 & 41.81 & $1.68-1041.72$ & 0.023 \\
\hline Stage, III/VI vs I/II & 3.86 & $0.78-19.16$ & 0.098 & 3.66 & $0.33-40.10$ & 0.288 \\
\hline Functionality, yes vs no & 1.37 & $0.34-5.49$ & 0.656 & 1.61 & $0.20-12.94$ & 0.656 \\
\hline
\end{tabular}

${ }^{*}$ Cox proportional analysis.

with PD events (17). Specifically, logistic regression was performed to evaluate the correlation between the dichotomized variable and PD event. The optimal cut off was determined as the point with the most significant split. An increase of CgA level greater than $40 \%$ of the CgA level in the follow-up after treatment may predict tumor progression (Table 5). Patients with a change of CgA level greater than a $40 \%$ increase had a higher risk of tumor progression or recurrence than those with a change of CgA level less than a $40 \%$ increase $(\mathrm{OR}=3.22,95 \% \mathrm{CI}: 1.11-9.34$ in the univariate analysis; $\mathrm{OR}=5.04,95 \% \mathrm{CI}: 1.31-19.4$ in the multivariate analysis, adjusted for the age of diagnosis, grade, stage, functionality, and sex).

\section{DISCUSSION}

Our study showed that high baseline CgA levels were associated with advanced stage in GEP-NETs. High baseline CgA levels and G2 were independent factors to predict the poor OS for GEPNET patients. A $40 \%$ or greater increase of CgA level during follow-up might be a predictive factor for tumor progression or recurrence of GEP-NETs.

CgA level has been shown to be elevated in various diseases, including benign and malignant diseases. Mild elevation of $\mathrm{CgA}$ level has been shown in a variety of systemic diseases, such as

TABLE 5 | Logistic regression analysis for odds ratio of tumor progression by ratio of change of CgA level, sex, age, grade, stage, and functionality.

\begin{tabular}{|c|c|c|c|c|c|c|}
\hline & \multicolumn{3}{|c|}{ Univariate } & \multicolumn{3}{|c|}{ Multivariate } \\
\hline & OR & $95 \% \mathrm{Cl}$ & $P$ value & OR & $95 \% \mathrm{Cl}$ & $P$ value \\
\hline Ratio of change of CgA level, $\geq 0.4$ vs $<0.4$ & 3.22 & $1.11-9.34$ & 0.031 & 5.04 & $1.31-19.4$ & 0.019 \\
\hline Sex, men vs women & 1.59 & $0.64-3.93$ & 0.315 & 0.89 & $0.28-2.87$ & 0.843 \\
\hline Age of diagnosis & 0.98 & $0.95-1.01$ & 0.217 & 1 & 0.96-1.04 & 0.950 \\
\hline Grade, G2 vs G1 & 8.4 & $3.02-23.34$ & $<0.001$ & 7.44 & $2.19-25.28$ & 0.001 \\
\hline Stage, III/IV vs I/II & 5 & $1.92-13.0$ & 0.001 & 3.15 & $0.96-10.31$ & 0.058 \\
\hline Functionality, yes vs no & 0.82 & $0.3-2.25$ & 0.704 & 0.49 & 0.13-1.82 & 0.288 \\
\hline
\end{tabular}


cardiovascular disease (hypertension, congestive heart failure, myocardial infarction), renal disease (renal failure), liver disease (liver dysfunction, liver cirrhosis), lung disease (chronic obstructive pulmonary disease), inflammatory diseases (inflammatory bowel disease, rheumatoid arthritis), and sepsis (8). Mild elevation of CgA has also been detected in some benign or malignant cancers, such as parathyroid adenoma, thyroid cancer, hepatocellular carcinoma, and lung cancer (small cell carcinoma and non-small cell carcinoma) (8). Marked elevation of $\mathrm{CgA}$ was noted in neuroendocrine tumors (8). Elevation of CgA can also be related to acid suppressive medications (9). The non-oncologic and nonNET oncologic conditions impair the specificity of CgA for the diagnosis or the prognosis of NETs. NETest, a PCR-based 51transcript signature for NETs, has been shown to have a higher sensitivity and specificity than CgA for the detection of NETs or prediction of tumor progression (18-20). However, it is still not routinely used in clinical practice probably due to cost and technical concerns. Although the specificity of $\mathrm{CgA}$ is not good, $\mathrm{CgA}$ is the most common non-specific circulating biomarker used for the follow-up of neuroendocrine tumors.

The sensitivity of $\mathrm{CgA}$ was reported to be related to the functionality and the extent of the NETs. Nehar et al. have shown that the sensitivity in patients with secreting tumors and nonsecreting tumors was $73 \%$ and $45 \%$, respectively $(\mathrm{P}<0.004)$ at the cut off value of $130 \mathrm{ng} / \mathrm{ml}$ of CgA. Significantly higher levels of CgA were noted in patients with metastatic disease $(3444 \pm 16256$ $\mathrm{ng} / \mathrm{ml})$ than those without $(174 \pm 233 \mathrm{ng} / \mathrm{ml}, P<0.001)(11)$. Campana et al. have also shown that CgA levels were higher in NET patients than those with chronic active gastritis or healthy participants (12). Higher levels of CgA were observed in NET patients with diffuse disease compared to those with local or hepatic disease, and those that were disease-free (12). Jason et al. have shown that the CgA levels were significantly higher in GEPNET patients with more than five liver metastases than those with fewer than five liver metastases or lymph node metastases (10). Advanced age and a CgA level greater than $5000 \mathrm{ng} / \mathrm{ml}$ were independent prognostic factors for worse OS in midgut NETs (10). Raoof et al. have reported that $79 \%$ of 445 small pancreatic NET $(\leq 2 \mathrm{~cm}$ ) patients were categorized with low CgA at a cut off value of $420 \mathrm{ng} / \mathrm{ml}$ (21). In our study, 21 of the 27 stage I patients (77.8\%) had low baseline CgA levels whereas 12 of the 22 stage IV patients (54.5\%) had low baseline CgA levels. Our result also showed an association between the baseline CgA levels and the extent of NETs and this is compatible with the findings of the previous studies. However, we did not observe any differences in the baseline CgA levels in GEP-NETs by grade (G1 vs G2), functionality, or primary site (pancreas vs non-pancreas) in our study. Different from other studies, we observed that lower baseline CgA levels were noted in men (75\%) than in women (50\%, $P=0.045)$.

The CgA level was also reported to be associated with the prognosis of NET patients. Yao et al. analyzed and reported the prognostic role of $\mathrm{CgA}$ and NSE in patients with low- to intermediate-grade advanced pancreatic NET from the RADIANT-1 phase II study. Elevated baseline CgA levels were associated with a shorter progression-free survival (PFS, 8.34 months in the elevated CgA group vs 15.64 months in the nonelevated CgA group, $P=0.03$ ) and OS (16.95 months vs not reached, $P<0.001)$. The prognostic role was also observed in NSE (14). Yao et al. also evaluated the impact of several biomarkers (baseline levels of CgA, NSE, and multiple soluble angiogenetic biomarkers) on the OS of advanced, progressive, low- or intermediate-grade pancreatic NET patients who received everolimus or placebo in the RADIANT-3 trial. The lower baselines of CgA, NSE, placental growth factor (PIGF), and soluble vascular endothelial growth factors 1 were associated with better OS. However, only NSE and PIGF remained significantly associated with $O S$ in the multivariate analysis. The effect of $\mathrm{CgA}$ was borderline significant $(\mathrm{HR}=0.76,95 \%$ CI, $0.57-1, P=0.05$ ) in the multivariate analysis (13). Ahmed et al. analyzed the data of 360 patients with midgut NETs with liver metastases from the UKI NET group. They reported that increasing age at diagnosis, higher Ki-67, increasing urinary hydroxyindole acetic acid levels, higher CgA levels, high tumor volume, and resection of primary tumor were associated with a worse OS in the univariate analysis (22). However, only age, Ki67 , and resection of primary tumor were identified as the independent predictors of survival in multivariate analysis (22). Chou et al. have reported that Eastern Cooperative Oncology Groups performance score 0-1, G1-2, single organ metastasis, and baseline CgA level less than twice the upper normal range were independent prognostic factors for OS of advanced GEPNET patients (15). Most studies demonstrated the prognostic role of $\mathrm{CgA}$ in advanced GEP-NETs whereas some studies showed no significant role of $\mathrm{CgA}$ as the predictor for OS. Fewer studies analyzed the prognostic role of $\mathrm{CgA}$ level in early-stage cases. Raoof et al. showed that CgA level (high vs low at a cut off value of $420 \mathrm{ng} / \mathrm{ml}$ ) was an independent predictive factor for OS in small pancreatic NET (tumor $\leq 2$ $\mathrm{cm}$ ) patients in multivariate analysis after adjusting for tumor size, grade, nodal status, and academic status of the facility (HR=7.9, 95\% CI, 2.34-26.69, $P=0.001)$ (21). They also observed that the OS was not significantly different between patients with low CgA levels receiving or not receiving tumor resection. But the OS was worse for the patients with high CgA levels who had not received tumor resection than those who had received tumor resection. ${ }^{21}$ In our study, we observed that the patients with low baseline CgA levels had significantly better OS than those with high baseline CgA levels. The significance persisted in multivariate analysis after adjusting for age, sex, grade, stage, and functionality. The result supported the prognostic role of baseline CgA levels in GEP-NETs, including early-stage and advanced-stage patients.

Circulating CgA during the follow-up of GEP-NET patients receiving systemic treatment or surveillance has also been investigated. Yao et al. reported that early $\mathrm{CgA}$ and NSE response ( $\geq 30 \%$ decrease of CgA or NSE from baseline or normalization at week 4) were predictors for longer PFS and OS in advanced pancreatic NET patients receiving everolimus treatment (RADIANT-1 study). The median PFS for the patients with early CgA response was 13.31 months whereas the median PFS for those without early response was only 7.52 
months $(P<0.001)$. The median OS for the patients with an early CgA response was 24.9 months whereas the median OS for those without an early CgA response was 12.71 months $(P=0.01)(14)$. Jensen et al. retrospectively analyzed the change of CgA level during treatment for CgA-producing ileo-cecal NET patients. They demonstrated a cut off of $25 \%$ for the prediction of tumor response after treatment with a $25 \%$ or greater increase predicting tumor progression and a $25 \%$ or greater decrease predicting tumor regression (23). Chou et al. also analyzed the change of CgA level and tumor response for advanced GEP-NET patients during treatment. They demonstrated that a change of CgA level $>17 \%$ distinguished partial response and stable disease from progressive disease with a sensitivity and specificity of $91.2 \%$ and $82.9 \%$, respectively (15). However, some studies did not validate $\mathrm{CgA}$ as a surrogate marker for tumor progression of NETs. Vezzosi et al. prospectively analyzed the concordance between CgA variation and RECIST criteria for tumor response in 39 metastatic well-differentiated GEP-NET patients. They showed that change of CgA at the 6-month follow-up ( $\geq 25 \%$ increase versus $<25 \%$ increase) had a sensitivity and a specificity of $71 \%$ and $50 \%$, respectively, for changes of tumor burden. The study did not validate $\mathrm{CgA}$ as a surrogate marker of tumor progression (24). Dam et al. prospectively monitored CgA levels for GEP-NET patients with metastasis or residual tumors and analyzed the predictive role of change of CgA level for tumor progression and regression. They reported an overall Spearman's rank correlation coefficient of $0.17(P=0.003)$ by analyzing the "matching pairs" of CgA and CT/MRI assessment. The diagnostic sensitivity and specificity of an increased CgA level for tumor progression were $36 \%$ and $82 \%$, respectively. The diagnostic sensitivity and specificity of a decreased CgA level for tumor regression were $79 \%$ and $69 \%$, respectively. They concluded a weak association between change of CgA and change in tumor burden (25). In our study, we observed that the patients with a $40 \%$ or greater increase of $\mathrm{CgA}$ during treatment or surveillance had a higher risk of developing tumor progression than those with less than a $40 \%$ increase of CgA during follow-up with an OR of 5.04 (95\% CI, 1.31-19.4, $P=0.019$ ) by multivariate logistic regression. The result supported the predictive role of change of CgA level for tumor progression in advanced GEP-NETs. Our patient population included early and advanced stages. The results suggested that an increase of $\mathrm{CgA}$ may also predict recurrence of early-stage GEP-NET after complete resection.

This is a retrospective study and the frequency and timing of measurement and follow-up of CgA level and tumor response varied according to their disease status and treatment. Therefore, we could not use repeated measurements of $\mathrm{CgA}$ to evaluate longitudinal change of $\mathrm{CgA}$ for tumor response prediction. However, we used cross-sectional analysis for the change of $\mathrm{CgA}$ to predict tumor progression that was not interfered with by

\section{REFERENCES}

1. Yao JC, Hassan M, Phan A, Dagohoy C, Leary C, Mares JE, et al. One Hundred Years After "Carcinoid": Epidemiology of and Prognostic Factors the fluctuation of CgA level during serial follow-up. Furthermore, to gain acceptance as a clinically meaningful observation, the change of $\mathrm{CgA}$ level would require testing in multi-center clinical therapeutic trials. Most importantly, the method of matching should be performed to reduce or eliminate the effects of confounding.

\section{CONCLUSIONS}

Our results suggested that baseline CgA level is associated with the disease extent and OS of GEP-NET patients. A $40 \%$ or greater increase of change of CgA level may predict tumor progression or recurrence during treatment or surveillance of GEP-NETs.

\section{DATA AVAILABILITY STATEMENT}

The datasets presented in this article are not readily available because the dataset is not available due to IRB restriction. Requests to access the datasets should be directed to Y-SS, ysshan@ncku.edu.tw.

\section{ETHICS STATEMENT}

The studies involving human participants were reviewed and approved by the Institutional Review Board of National Cheng Kung University Hospital. Written informed consent for participation was not required for this study in accordance with the national legislation and the institutional requirements.

\section{AUTHOR CONTRIBUTIONS}

H-JT had acquisition of data, analyzed and interpreted data, and wrote and revised the manuscript. $\mathrm{C}-\mathrm{FH}$ analyzed and interpreted the data and wrote and revised the manuscript. JC analyzed and interpreted the data and revised the manuscript. L-TC, Y-JC, and C-JY analyzed and interpreted the data and revised the manuscript. Y-SS designed the study, analyzed and interpreted the data and revised the manuscript. All authors had final approval of the version of the manuscript to be submitted.

\section{ACKNOWLEDGMENTS}

We thank Norvatis for their support during CgA measurement.

for Neuroendocrine Tumors in 35,825 Cases in the United States. J Clin Oncol (2008) 26(18):3063-72. doi: 10.1200/JCO.200701504377

2. Hauso O, Gustafsson BI, Kidd M, Waldum HL, Drozdov I, Chan AKC, et al. Neuroendocrine Tumor Epidemiology: Contrasting Norway and North America. Cancer (2008) 113(10):2655-64. doi: 10.1002/cncr.23883 
3. Tsai HJ, Wu CC, Tsai CR, Lin SF, Chen LT, Chang JS. The Epidemiology of Neuroendocrine Tumors in Taiwan: A Nation-Wide Cancer Registry-Based Study. PLoS One (2013) 8(4):e62487. doi: 10.1371/journal.pone.0062487

4. Dasari A, Shen C, Halperin D, Zhao B, Zhou S, Xu Y, et al. Trends in the Incidence, Prevalence, and Survival Outcomes in Patients With Neuroendocrine Tumors in the United States. JAMA Oncol (2017) 3 (10):1335-42. doi: 10.1001/jamaoncol.2017.0589

5. Chang JS, Chen LT, Shan YS, Chu PY, Tsai CR, Tsai HJ. An Updated Analysis of the Epidemiologic Trends of Neuroendocrine Tumors in Taiwan. Sci Rep (2021) 11(1):7881. doi: 10.1038/s41598-021-86839-2

6. Sansone A, Lauretta R, Vottari S, Chiefari A, Barnabei A, Romanelli F, et al. Specific and non-Specific Biomarkers in Neuroendocrine Gastroenteropancreatic Tumors. Cancers (2019) 11(8):1113. doi: 10.3390/ cancers 11081113

7. Loh YP, Cheng Y, Mahata SK, Corti A, Tota B. Chromogranin A and Derived Peptides in Health and Disease. J Mol Neurosci (2012) 48(2):347-56. doi: 10.1007/s12031-012-9728-2

8. Mahata SK, Corti A. Chromogranin A and Its Fragments in Cardiovascular, Immunometabolic, and Cancer Regulation. Ann NY Acad Sci (2019) 1455 (1):34-58. doi: 10.1111/nyas.14249

9. Marotta V, Zatelli MC, Sciammarella C, Ambrosio MR, Bondanelli M, Colao A, et al. Chromogranin A as Circulating Marker for Diagnosis and Management of Neuroendocrine Neoplasms: More Flaws Than Fame. Endocr Relat Cancer (2018) 25(1):R11-29. doi: 10.1530/ERC-17-0269

10. Janson ET, Holmberg L, Stridsberg M, Eriksson B, Theodorsson E, Wilander E, et al. Carcinoid Tumors: Analysis of Prognostic Factors and Survival in 301 Patients From a Referral Center. Ann Oncol (1997) 8(7):685-90. doi: 10.1023/ a:1008215730767

11. Nehar D, Lombard-Bohas C, Olivieri S, Claustrat B, Chayvialle JA, Penes MC, et al. Interest of Chromogranin A for Diagnosis and Follow-Up of Endocrine Tumours. Clin Endocrinol (2004) 60(5):644-52. doi: 10.1111/j.13652265.2004.02030.x

12. Campana D, Nori F, Piscitelli L, Morselli-Labate AM, Pezzilli R, Corinaldesi R, et al. Chromogranin A: Is It a Useful Marker of Neuroendocrine Tumors. J Clin Oncol (2007) 25(15):1967-73. doi: 10.1200/JCO.2006.10.1535

13. Yao JC, Pavel M, Lombard-Bohas C, Van Cutsem E, Voi M, Brandt U, et al. Everolimus for the Treatment of Advanced Pancreatic Neuroendocrine Tumors: Overall Survival and Circulating Biomarkers From the Randomized, Phase III RADIANT-3 Study. J Clin Oncol (2016) 34 (32):3906-13. doi: 10.1200/JCO.2016.68.0702

14. Yao JC, Pavel M, Phan AT, Hoosen S, St Peter J, Cherfi A, et al. Chromogranin A and Neuron-Specific Enolase as Prognostic Markers in Patients With Advanced pNET Treated With Everolimus. J Clin Endocrinol Metab (2011) 96(12):3741-9. doi: 10.1210/jc.2011-0666

15. Chou WC, Chen JS, Hung YS, Hsu JT, Chen TC, Sun CF, et al. Plasma Chromogranin A Levels Predict Survival and Tumor Response in Patients With Advanced Gastroenteropancreatic Neuroendocrine Tumors. Anticancer Res (2014) 34(10):5661-70.

16. Chou WC, Hung YS, Hsu JT, Chen JS, Lu CH, Hwang TL, et al. Chromgranin A Is a Reliable Biomarker for Gastroenteropancreatic Neuroendocrine Tumors in an Asian Population of Patients. Neuroendocrinology (2012) 95 (4):344-50. doi: 10.1159/000333853

17. Budczies J, Klauschen F, Sinn BV, Győrffy B, Schmitt WD, Darb-Esfahani S, et al. Cutoff Finder: A Comprehensive and Straightforward Web Application
Enabling Rapid Biomarker Cutoff Optimization. PLoS One (2012) 7:e51862. doi: 10.1371/journal.pone.0051862

18. Modlin IM, Drozdov I, Alaimo D, Callahan S, Teixiera N, Bodei L, et al. A Multianalyte PCR Blood Test Outperforms Single Analyte ELISAs (Chromogranin A, Pancreastatin, Neurokinin A) for Neuroendocrine Tumor Detection. Endocr Relat Cancer (2014) 21(4):615-28. doi: 10.1530/ ERC-14-0190

19. Pavel M, Jann H, Prasad V, Drozdov I, Modlin IM, Kidd M. NET Blood Transcript Analysis Defines the Crossing of the Clinical Rubicon: When Stable Disease Becomes Progressive. Neuroendocrinology (2017) 104(2):170 82. doi: $10.1159 / 000446025$

20. Malczewska A, Kos-Kudla B, Kidd M, Drozdov I, Bodei L, Matar S, et al. The Clinical Application of a Multigene Liquid Biopsy (NETest) in Neuroendocrine Tumors. Adv Med Sci (2020) 65(1):18-29. doi: 10.1016/ j.advms.2019.10.002

21. Raoof M, Jutric Z, Melstrom LG, Lee B, Li D, Warner SG, et al. Prognostic Significance of Chromogranin A in Small Pancreatic Pancreatic Neuroendocrine Tumors. Surgery (2019) 165(4):760-6. doi: 10.1016/ j.surg.2018.10.018

22. Ahmed A, Turner G, King B, Jones L, Culliford D, McCance D, et al. Midgut Neuroendocrine Tumours With Liver Metastases: Results of the UKINETS Study. Endocr Relat Cancer (2009) 16(3):885-94. doi: 10.1677/ERC-09-0042

23. Jensen HK, Hilsted L, Jensen C, Mynster T, Rehfeld JF, Knigge U. Chromogranin A Is a Sensitive Marker of Progression or Regression in Ileo-Cecal Neuroendocrine Tumors. Scand J Gastroenterol (2013) 48(1):707. doi: 10.3109/00365521.2012.733953

24. Vezzosi D, Walter T, Laplanche A, Raoul JL, Dromain C, Ruszniewski P, et al. Chromogranin A Measurement in Metastatic Well-Differentiated Gastroenteropancreatic Neuroendocrine Carcinoma: Screening for False Positive and a Prospective Follow-Up Study. Int J Biol Markers (2011) 26 (2):94-101. doi: 10.5301/JBM.2011.8327

25. Dam G, Grønbæk H, Sorbye H, Evensen ET, Paulsson B, Sundin A, et al. Prospective Study of Chromogranin A as a Predictor of Progression in Patients With Pancreatic, Small-Intestinal, and Unknown Primary Neuroendocrine Tumors. Neuroendocrinology (2020) 110(3-4):217-24. doi: $10.1159 / 000503833$

Conflict of Interest: The authors declare that the research was conducted in the absence of any commercial or financial relationships that could be construed as a potential conflict of interest.

Publisher's Note: All claims expressed in this article are solely those of the authors and do not necessarily represent those of their affiliated organizations, or those of the publisher, the editors and the reviewers. Any product that may be evaluated in this article, or claim that may be made by its manufacturer, is not guaranteed or endorsed by the publisher.

Copyright (C) 2021 Tsai, Hsiao, Chang, Chen, Chao, Yen and Shan. This is an openaccess article distributed under the terms of the Creative Commons Attribution License (CC BY). The use, distribution or reproduction in other forums is permitted, provided the original author(s) and the copyright owner(s) are credited and that the original publication in this journal is cited, in accordance with accepted academic practice. No use, distribution or reproduction is permitted which does not comply with these terms. 